\title{
Feasibility of a home-based exercise intervention with remote guidance for patients with stable grade II and III gliomas
}

\author{
a pilot randomized controlled trial
}

\author{
Author(s) \\ Gehring, Karin; Kloek, Corelien JJ; Aaronson, Neil K; Janssen, Kasper W; Jones, Lee W; \\ Sitskoorn, Margriet M; Stuiver, Martijn M
}

DOI

$10.1177 / 0269215517728326$

Publication date

2017

Document Version

Author accepted manuscript (AAM)

Published in

Clinical Rehabilitation

\section{License \\ CC BY}

Link to publication

\section{Citation for published version (APA):}

Gehring, K., Kloek, C. JJ., Aaronson, N. K., Janssen, K. W., Jones, L. W., Sitskoorn, M. M., \& Stuiver, M. M. (2017). Feasibility of a homebased exercise intervention with remote guidance for patients with stable grade II and III gliomas: a pilot randomized controlled trial. Clinical Rehabilitation. https://doi.org/10.1177/0269215517728326

If you believe that digital publication of certain material infringes any of your rights or (privacy) interests, please let the Library know, stating your reasons. In case of a legitimate complaint, the Library will make the material inaccessible and/or remove it from the website. Please contact the library:

https://www.amsterdamuas.com/library/contact/questions, or send a letter to: University Library (Library of the University of Amsterdam and Amsterdam University of Applied Sciences), Secretariat, Singel 425, 1012 WP Amsterdam, The Netherlands. You will be contacted as soon as possible.

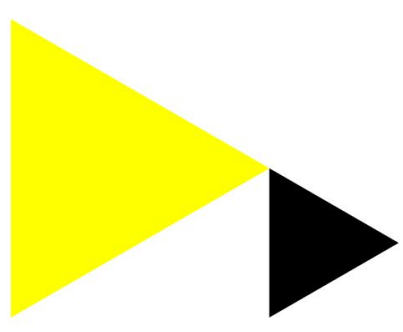




\title{
Feasibility of a home-based exercise intervention with remote guidance for patients with stable grade II and III gliomas: a pilot randomized controlled trial
}

\author{
Karin Gehring ${ }^{1,2}$, Corelien JJ Kloek ${ }^{1,3}$, Neil K Aaronson 4 , \\ Kasper W Janssen ${ }^{5}$, Lee W Jones ${ }^{6}$, Margriet M Sitskoorn' \\ and Martijn M Stuiver ${ }^{7,8}$
}

\begin{abstract}
Objective: In this pilot study, we investigated the feasibility of a home-based, remotely guided exercise intervention for patients with gliomas.

Design: Pilot randomized controlled trial (RCT) with randomization (2:I) to exercise or control group. Subjects: Patients with stable grade II and III gliomas.

Intervention: The six-month intervention included three home-based exercise sessions per week at $60 \%-85 \%$ of maximum heart rate. Participants wore heart rate monitors connected to an online platform to record activities that were monitored weekly by the physiotherapist.

Main measures: Accrual, attrition, adherence, safety, satisfaction, patient-reported physical activity, $\mathrm{VO}_{2}$ peak (by maximal cardiopulmonary exercise testing) and body mass index (BMI) at baseline and at six-month follow-up.

Results: In all, 34 of 136 eligible patients (25\%) were randomized to exercise training $(N=23)$ or the control group $(N=I I)$, of whom 19 and 9 , respectively, underwent follow-up. Mean adherence to prescribed sessions was $79 \%$. Patients' experiences were positive. There were no adverse events. Compared to the control group, the exercise group showed larger improvements in absolute $\mathrm{VO}_{2}$ peak (+ I58.9 mL/min; $95 \% \mathrm{Cl}:-44.8$ to 362.5$)$ and $\mathrm{BMI}\left(-0.3 \mathrm{~kg} / \mathrm{m}^{2} ; 95 \% \mathrm{Cl}:-0.9\right.$ to 0.2$)$. The median increase
\end{abstract}

'Department of Cognitive Neuropsychology, Tilburg University, Tilburg, The Netherlands

${ }^{2}$ Department of Neurosurgery, Elisabeth-TweeSteden Hospital, Tilburg, The Netherlands

${ }^{3}$ Research Group of Innovation of Human Movement Care, University of Applied Sciences Utrecht, Utrecht, The Netherlands ${ }^{4}$ Division of Psychosocial Research and Epidemiology, The Netherlands Cancer Institute, Amsterdam, The Netherlands ${ }^{5}$ Department of Sports and Exercise Medicine, Gelderse Vallei Hospital, Ede, The Netherlands

${ }^{6}$ Department of Medicine, Memorial Sloan Kettering Cancer Center, New York, NY, USA

\footnotetext{
7Department of Physical Therapy, The Netherlands Cancer Institute, Amsterdam, The Netherlands

${ }^{8}$ ACHIEVE, Faculty of Health, University of Applied Sciences Amsterdam, Amsterdam, The Netherlands

Corresponding author:

Karin Gehring, Department of Cognitive Neuropsychology, Tilburg University, Room S219, PO Box 90153, 5000 LE Tilburg, The Netherlands.

Email: k.gehring@uvt.nl
} 
in physical activity was 1489 metabolic equivalent of task (MET) minutes higher in the exercise group. The most reported reasons for non-participation were lack of motivation or time.

Conclusion: This innovative and intensive home-based exercise intervention was feasible in a small subset of patients with stable gliomas who were interested in exercising. The observed effects suggest that the programme may improve cardiorespiratory fitness. These results support the need for large-scale trials of exercise interventions in brain tumour patients.

\section{Keywords}

Glioma, brain tumour, exercise, physical training, physical fitness

Date received: 12 April 2017; accepted: 26 July 2017

\section{Introduction}

After treatment with surgery, radiotherapy and/or chemotherapy, patients with gliomas with favourable prognosis may live free from severe neurological symptoms for years until the disease progresses. During this period of time, many patients may suffer from a wide range of physical, psychological/emotional and cognitive symptoms. ${ }^{1}$ Few efforts have been undertaken to address these problems, despite the relatively young age and favourable prognosis of this clinical population.

Recently, Cormie et al. ${ }^{2}$ concluded, based on the evidence for beneficial effects of exercise in managing physical, psychological and cognitive symptoms in other populations, that exercise may be a promising intervention to aid in the management of the multitude of brain cancer symptoms and treatment side-effects. However, there has been very limited research on the feasibility and effectiveness of exercise interventions in patients with brain tumours. Feasibility may be a particular concern in this population, as neuro-oncological symptoms may limit patients' willingness and ability to participate in an exercise intervention (study).

To date, two small, uncontrolled (pilot) exercise intervention studies (respectively, $n=8$ and 14 at follow-up) have been conducted in this population, the results of which suggest that an exercise programme is both feasible and safe..$^{2,3}$ In a study on exercise preferences of 106 patients with brain tumours ( $50 \%$ glioma), the majority of the respondents were interested in physical exercise and felt that they would be able to do exercise. ${ }^{4}$ Glioma patients also reported a strong preference for home-based exercise programmes. ${ }^{4,5}$ Homebased interventions may be particularly important for brain cancer patients, as neurological symptoms such as epilepsy can restrict patients' mobility (i.e. the ability to drive or use public transportation). ${ }^{4}$

Recently, we developed and pilot-tested a novel home-based aerobic exercise intervention for patients with stable, lower-grade glioma. The ultimate aim of the programme was to maintain or improve cognitive function through improvement of aerobic fitness. ${ }^{6-8}$

The primary purpose of this article is to present a detailed evaluation of the six-month exercise intervention in terms of accrual, attrition, adherence, safety and patient satisfaction in a research setting. These results may help to design future studies on the benefits of exercise in brain tumour patients. In addition, we describe the direct effects of the programme on cardiorespiratory fitness $\left(\mathrm{VO}_{2}\right.$ peak) and self-reported physical activity, as we consider these intermediate endpoints as a measure of feasibility of an exercise programme designed to improve cognitive function. Detailed findings with respect to cognitive performance and patient-reported outcomes will be reported in a subsequent paper. 


\section{Methods}

\section{Study design}

Ethical approval was obtained from the medical ethics committee (METC) Brabant, Tilburg, The Netherlands (N44024.008.13). The trial was registered with www.clinicaltrials.gov: NCT02303938. Enrolment started in September 2013 and ended in December 2014.

In this pilot, randomized controlled study (RCT), patients were allocated to a six-month exercise intervention and a waiting-list control group.

\section{Participants}

We recruited patients from three Dutch hospitals: Elisabeth-TweeSteden (Elisabeth-TweeSteden Hospital) Hospital Tilburg, Haaglanden Medical Center The Hague and Erasmus Medical Center Rotterdam. Eligibility criteria were as follows: histologically proven or presumed (on the basis of clinical and magnetic resonance imaging (MRI) data) diffuse, low-grade (i.e. World Health Organization (WHO) grade II) glioma, or anaplastic glioma (WHO grade III); clinically stable for a minimum of six months prior to study entry as determined by MRI; current self-reported inactivity or only a moderate level of physical activity (i.e. $<20$ minutes of vigorous exercise on at least three days of the week) as assessed with the Physician-based Assessment and Counseling for Exercise (PACE); ${ }^{9}$ access to the Internet; basic fluency in the Dutch language; interest in undergoing the physical exercise programme under investigation; and, finally, a $\mathrm{VO}_{2}$ peak, as assessed with maximal cardiopulmonary exercise test, within the range of sedentary or recreationally active reference groups, ${ }^{10}$ allowing room for further improvement of fitness.

Exclusion criteria were as follows: antitumour treatment (i.e. surgery, radiotherapy, chemotherapy, corticosteroids) within six months prior to study entry; use of beta-blockers; ${ }^{11}$ psychiatric or severe cognitive problems that would preclude programme participation; serious orthopaedic conditions, motor deficits, cardiovascular, cardiopulmonary or neurological condition; or contra-indications for exercise without face-to face supervision as assessed with the Physical Activity Readiness Questionnaire
(PAR-Q) ${ }^{12}$ or as judged by the sports physician based on the cardiopulmonary exercise test; and no room for cognitive improvement, as assessed with neuropsychological testing.

\section{Recruitment procedure}

Potential participants underwent three screening phases. In the first screening phase, we identified potentially eligible patients via pathology databases or direct referral from the participating hospitals. Medically eligible patients received a study information letter from their physician and a reply card on which they could indicate whether they gave permission to be approached by phone. Interested patients were in this second phase called by a member of the research team who explained the study purpose and procedures, and screened for initial eligibility. In the third phase, all individual eligible patients provided informed consent, underwent neuropsychological testing and completed self-report questionnaires on cognitive symptoms, fatigue, sleep, mood and quality life at home. Subsequently, they were invited to undergo a maximal cardiopulmonary exercise test in a sports medical centre.

Eligible patients were randomly allocated in a 2:1 ratio to an exercise group or a waiting-list control group. A minimization procedure ${ }^{13}$ was used to ensure that the two groups were balanced on age $(<40,40-50,>50)$, education (lower vs. higher), WHO tumour grade (II vs. III), disease duration ( $<$ five vs. $\geq$ five years), relative $\mathrm{VO}_{2}$ classification according to reference groups (recreational physical activity vs. sedentary ${ }^{10}$ ) and performance on the letter digit substitution $\operatorname{task}^{14}(\leq 43$ vs. $>43)$. Allocation concealment was ensured using an online computer software program. ${ }^{15}$ All patients were informed about their allocation by phone. Besides the sports physicians who administered the exercise tests, the other assessors, nor the patients, could be blinded to group allocation.

The decision to halt participation in case of progressive disease during the course of the study depended on further treatment and/or was left up to the individual patient and his or her treating physician. 


\section{Exercise group}

The intervention comprised three home-based aerobic training sessions per week, for a duration of six months. At the start of the intervention, a physiotherapist visited participants at home. Patients received an individualized exercise prescription, based on their level of aerobic fitness, to exercise at $60 \%-85 \%$ of their maximum heart rate (see Appendix for a more elaborate description and build-up of the activities). They could choose one or more central activities, as long as these could meet the prescribed exercise intensity. The physiotherapist instructed patients in how to use a sports watch with a heart rate monitor, ${ }^{16}$ and how to upload training data at least once a week. During the activities, this watch provided immediate feedback about heart rate and, if applicable, speed and distance. Patients kept a log of their training experiences. The physiotherapist monitored the training data on the platform on a weekly basis and provided additional personal feedback by e-mail. In case of motivational problems, fatigue, injury or technical problems, more frequent e-mail/phone contact was allowed. After the final exercise test, participants were called a last time to discuss the programme and exercise test results and to discuss continuation of physical exercise after the study period.

\section{Waiting-list control group}

Patients in the waiting-list control group were advised to maintain an active lifestyle, in accordance with Dutch public health guidelines, ${ }^{17}$ which were described in two motivational brochures. Patients in this group also received bi-monthly phone calls from the research assistant during which general questions about their health were asked. These calls were intended to provide some control for potential effects due to the attention that was given to the exercise group. After all assessments had been completed, participants in the control group were offered a training watch and a general exercise prescription.

\section{Outcome measures}

Baseline assessments of physical fitness, patientreported outcomes and cognitive function were conducted before randomization (T0) and follow-up assessments at six months, after patients in the exercise group had completed their training (T1).

Sociodemographic and clinical information. Patients' age, sex and level of education were obtained via interview at baseline. Clinical information, including date of diagnosis and tumour characteristics, such as site, and histology, and anticancer and antiepileptic treatment was abstracted from the medical records.

Feasibility parameters. Accrual was defined as the percentage of eligible patients who entered into the study. Attrition was defined as the percentage of randomized patients who dropped out of the study. Reasons for non-participation and discontinuation were recorded.

Individual exercise data on type of sport, duration of intervention, attended training days, mean session duration (minutes) and mean intensity (\% of maximum heart rate) were extracted from the patients' accounts on the online platform. Adherence was then calculated as the percentage of the physical exercise sessions completed out of prescribed sessions in the period in which a patient participated in the programme; $\geq 75 \%$ was considered sufficient. Average intensity was indicated by the average heart rate of all training sessions as a percentage of the maximum heart rate as measured during the first exercise test; a percentage of $60 \%-$ $85 \%$ was considered sufficient.

Finally, two physiotherapists (C.J.J.K. and M.S.) independently rated overall exercise performance for each participant as A (excellent/ good), B (adequate) or C (inadequate), based on observed adherence, session duration and intensity. Disagreements were resolved by consensus. Injuries or side-effects attributable to the exercise intervention were reported in the physiotherapists' logbook.

Patients' satisfaction. Patients' satisfaction with the programme was assessed in the exercise group with a post-training, study-specific questionnaire. Qualitative data from patients' e-mails to the physiotherapist were collected. 
Self-reported physical activity. Self-reported level of physical activity was assessed by calculating metabolic equivalent of task (MET) minutes/week acquired during walking, moderate or vigorous physical activity, as self-reported on the International Physical Activity Questionnaire (IPAQ). ${ }^{18,19}$

Physical outcomes. Physical fitness was assessed at baseline (T0) and after six months (T1) in both study groups with a maximal cardiopulmonary exercise test on a cycle ergometer with electrocardiogram (ECG) and breathing gas analysis. The purpose of the test in this study was threefold: (1) safety testing and screening, (2) baseline assessment for use in individualizing exercise prescription and (3) (outcome) assessment of $\mathrm{VO}_{2}$ peak.

The exercise test was performed in one of the four participating Sports Medical Centers affiliated with the patient's hospital. These tests were supervised by different sports physicians, who followed a protocol based on the American College of Sports Medicine (ACSM) guidelines, ${ }^{20}$ and who were blinded to allocation. Raw output data of the exercise test were used to extract absolute $\mathrm{VO}_{2}$ peak data and calculate relative $\mathrm{VO}_{2}$ peak (absolute $\mathrm{VO}_{2}$ peak divided by body weight).

Neuropsychological assessments. Cognitive functioning was assessed at the patients' home by a battery of neuropsychological tests of attention, memory and executive function. ${ }^{21}$ Patients were also given several questionnaires on self-reported cognitive symptoms, fatigue, sleep, mood and quality life to be completed and returned by mail. For the purpose of this article, only the feasibility of administration of these assessments in this pilot study was evaluated.

\section{Statistical methods}

Based on historical patient census data from the three participating centres, we expected to be able to include 60 patients in the study. Due to the lack of preliminary data on the effect of exercise on cognitive function in glioma patients, there was no meaningful way to perform sample size calculations for this pilot feasibility study.
Baseline demographics, clinical and physical data were compared between the study groups, and between dropouts and patients who completed follow-up. Statistical analyses were conducted for group outcomes on absolute and relative $\mathrm{VO}_{2}$ peak, body mass index (BMI), weight and patientreported physical activity. Absolute $\mathrm{VO}_{2}$ peak was used as the primary outcome for analysis of changes in physical fitness. No a priori level of statistical significance was set, as the objective of the study was not to test hypotheses, but to estimate potential effect sizes. Ninety-five per cent confidence intervals were calculated, along with twosided $P$-values, for all comparisons.

First, we performed an intention-to-treat analysis to estimate the within-group changes in physical fitness, BMI, body weight and physical activity using paired-samples $t$-tests. If test assumptions were violated, a Wilcoxon signed-rank test was used, and a 95\% confidence interval for the median change was estimated via 1000 bootstraps.

In addition, to estimate the change specifically attributable to the intervention, we used linear regression analyses to compare the outcomes of both groups noted above at six months, controlling for baseline scores. Adjusted mean between-group differences are reported with a $95 \%$ confidence interval. In case of violated assumptions for these tests, a Mann-Whitney $U$-test (inevitably without correction for baseline score) was used, and presented with bootstrap $95 \%$ confidence intervals for the difference between the group medians. Cohen' ${ }^{22} d$ effect sizes were calculated for between-group effects, for which 0.2 is considered a 'small' effect, 0.5 a 'medium' effect and 0.8 and larger a 'large' effect.

SPSS version 22.0 was used for all statistical analyses, ${ }^{23}$ except the calculation of $95 \%$ confidence intervals for the difference between group medians, which was done in R 3.3.1.24

\section{Results}

\section{Accrual and attrition}

After the three phases of screening, 34 of 136 invited patients $(25 \%)$ were recruited and allocated to the intervention $(N=23)$ or the control $(N=11)$ group. 
Due to personnel and time (i.e. financial) constraints, we could not prolong the recruitment phase of the study, or include an additional participating centre, to recruit the projected 60 participants. Figure 1 displays the detailed information on recruitment and retention. In all, $30(22 \%)$ patients who did not participate did not provide a reason for declining, did not respond to the invitation or could not be reached by phone. Of the 62 patients for whom a reason for nonparticipation was known, the most reported reasons were lack of motivation or time to exercise. In all, 10 patients $(16 \%)$ reported being physically unable to undergo intensive training or assessments. Of the 70 patients who were screened by telephone and were interested in participating, $14(20 \%)$ already met the criteria for a physically active lifestyle based on the PACE questionnaire. One patient withdrew after the baseline exercise test, but before randomization, when she understood that the programme did not include face-to-face training with a physiotherapist.

At baseline, 29 patients $(85 \%)$ had a $\mathrm{VO}_{2}$ peak comparable to sedentary age-matched healthy controls and 5 (15\%) comparable to people involved in recreational sports. ${ }^{10}$ Except for a higher proportion of grade II tumours in the exercise group, the groups were comparable at baseline with respect to sociodemographic, clinical or physical characteristics (Table 1).

No differences were observed in baseline sociodemographic, clinical and physical characteristics between dropouts $(N=6)$ and patients who completed follow-up measurement $(N=28)$, except that patients with grade III gliomas were more likely to drop out. One patient who learned during the intervention that he had progressive disease was able to undergo the follow-up exercise test.

\section{Exercise programme data: exercise preferences and adherence}

A detailed overview of training variables per case in the exercise group is presented in the Online table. Most patients ( $n=15$ of 23) chose a combination of activities. Other patients chose a single type of exercise: indoor cycling $(n=3)$, outdoor cycling $(n=3)$, running/walking $(n=1)$ or swimming $(n=1)$. The majority $(n=19)$ of participants completed the full 24-week exercise programme (range: 23-25 weeks).
Mean adherence was 79\% (SD: 21\%; range: $39 \%-100 \%$; i.e. 2.4 sessions per week). A total of 16 patients $(70 \%)$ completed $\geq 75 \%$ of the prescribed training sessions and were classified as adherent although two dropped out before the sixmonth assessment. Reasons for non-adherence were lack of time, tiredness and fear of epileptic seizures, 'lack of self-discipline for home-based exercise', lack of motivation due to divorce, and medical advice not to swim due to ear problems (patient not interested in alternative activities). On average, patients exercised 126 minutes per (active) week (range $=26-322$ minutes). Average exercise intensity was $76 \%$ (range $=68 \%-87 \%$ ) of maximum heart rate. Overall, exercise performance was classified as excellent/good in 16 patients $(70 \%)$, adequate in $3(13 \%)$ and inadequate in $4(17 \%)$.

Four participants required additional assistance with using the heart rate monitor. These problems were resolved via consultation by telephone.

\section{Safety and injury}

After the baseline exercise test, one patient was excluded from further participation for safety reasons because of ECG deviations. The majority of exercise participants had (had) epileptic seizures (74\%) and used anti-epileptic drugs (61\%), but this did not complicate exercise adherence (see Online table). However, one patient, who had already experienced frequent seizures that continued during the exercise intervention, reported serious feelings of insecurity and doubts about her capability to continue the exercise intervention. The physiotherapist and the patient together decided to decrease the frequency of training session from three to two sessions per week.

There were no exercise-induced injuries although one patient reported aggravation of preexisting osteoarthritis-related knee pain at the sixth month of the exercise programme. The physiotherapists recommended reducing resistance during training and this decreased the pain symptoms.

\section{Patient-reported satisfaction}

Data on satisfaction were available for 20 patients. Of these, 16 patients (84\%) evaluated the physical 


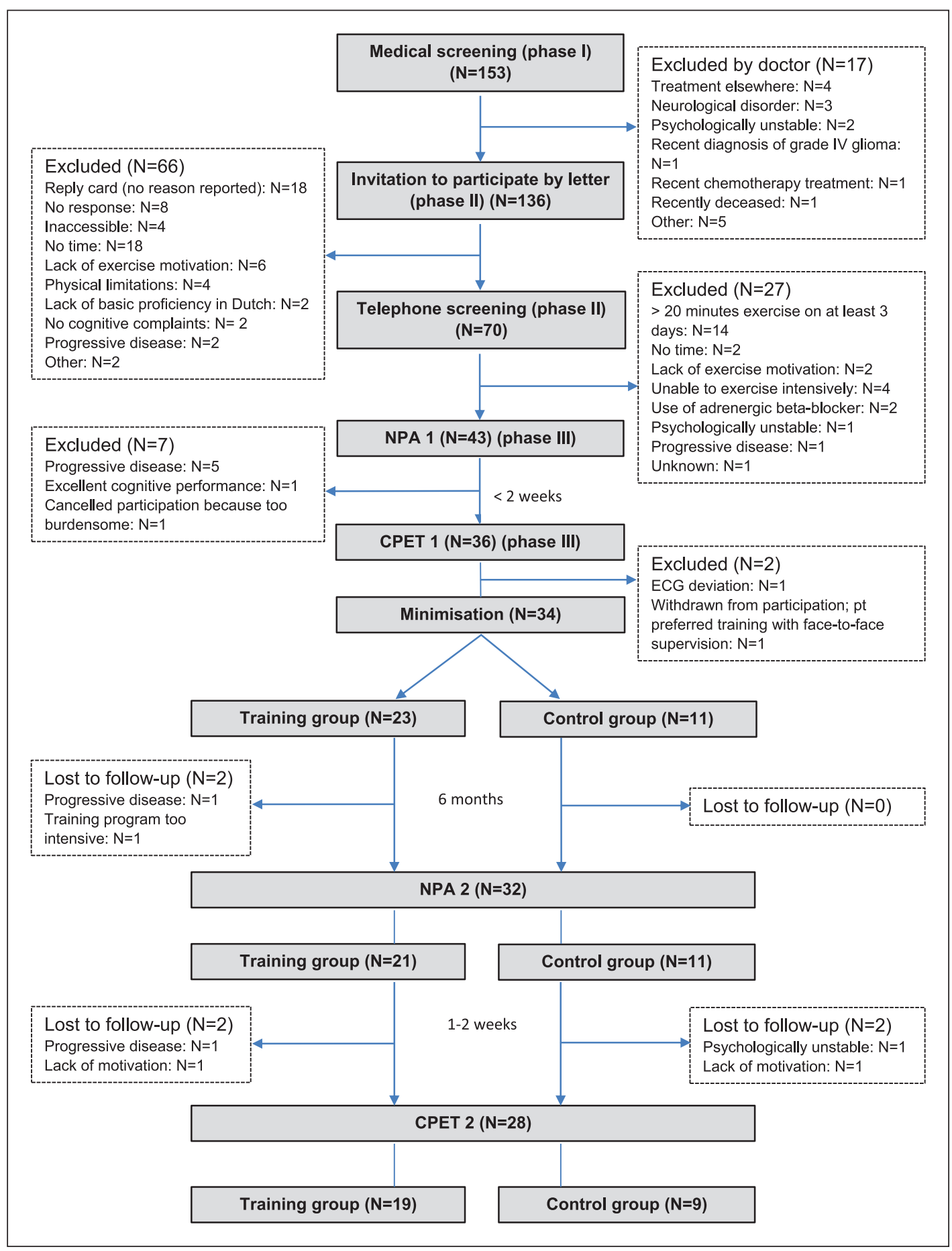

Figure I. Flow of participants through the trial (accrual and attrition).

NPA: neuropsychological assessment; CPET: cardiorespiratory exercise testing; ECG: electrocardiogram. 
Table I. Baseline sociodemographic, clinical and physical characteristics.

\begin{tabular}{|c|c|c|}
\hline Characteristic & Intervention group $(N=23)$ & Control group $(N=I I)$ \\
\hline \multicolumn{3}{|l|}{ Age, years } \\
\hline Mean (SD) & $48.0(9.4)$ & $48.0(11.9)$ \\
\hline Female, $N(\%)$ & $13(56 \%)$ & $6(55 \%)$ \\
\hline \multicolumn{3}{|l|}{ Education, $N(\%)$} \\
\hline Low & $2(9 \%)$ & $0(0 \%)$ \\
\hline Middle & $10(43 \%)$ & $6(55 \%)$ \\
\hline High & II (48\%) & $5(45 \%)$ \\
\hline \multicolumn{3}{|l|}{ WHO tumour grade, $N(\%)$} \\
\hline Grade II & $16(70 \%)$ & $6(55 \%)$ \\
\hline Grade III & 7 (30\%) & $5(45 \%)$ \\
\hline \multicolumn{3}{|l|}{ Tumour histology, N (\%) } \\
\hline Astrocytoma & $8(35 \%)$ & $5(46 \%)$ \\
\hline Oligodendroglioma & $12(52 \%)$ & $5(46 \%)$ \\
\hline Oligoastrocytoma & $3(13 \%)$ & I (8\%) \\
\hline \multicolumn{3}{|l|}{ Disease duration, years } \\
\hline Mean (SD) & $7.6(4.9)$ & $8.5(8.6)$ \\
\hline Left hemisphere, $N(\%)$ & $10(43 \%)$ & $4(36 \%)$ \\
\hline \multicolumn{3}{|l|}{ Surgery, $N(\%)$} \\
\hline No & 0 & I (9\%) \\
\hline Biopsy & $4(17 \%)$ & I (9\%) \\
\hline Resection & $19(83 \%)$ & $9(82 \%)$ \\
\hline Chemotherapy, N (\%) & $9(39 \%)$ & $4(36 \%)$ \\
\hline Radiotherapy, $N(\%)$ & 14 (6l\%) & $5(45 \%)$ \\
\hline Epilepsy & 17 (73.9\%) & $8(72.7 \%)$ \\
\hline Anti-epileptic drugs & 14 (60.9\%) & $6(54.5 \%)$ \\
\hline \multicolumn{3}{|l|}{$\mathrm{VO}_{2}$ peak absolute, $\mathrm{mL} / \mathrm{min}$} \\
\hline Mean (SD) & $2252.0(728.0)$ & $2181.5(741.0)$ \\
\hline \multicolumn{3}{|l|}{$\mathrm{VO}_{2}$ peak relative, $\mathrm{mL} / \mathrm{kg} / \mathrm{min}$} \\
\hline Mean (SD) & $26.5(7.4)$ & $25.9(4.8)$ \\
\hline \multicolumn{3}{|l|}{$\mathrm{VO}_{2}$ peak classification, $\mathrm{N}(\%)^{10}$} \\
\hline Recreational (vs. sedentary) & $4(17 \%)$ & I (9\%) \\
\hline \multicolumn{3}{|c|}{ Self-reported physical activity, MET min/week } \\
\hline Median (25th; 75th percentile) & $4399.5(1468.0 ; 8640.0)$ & $4583.5(1838.0 ; 13,457.0)$ \\
\hline \multicolumn{3}{|l|}{ Body weight, $\mathrm{kg}$} \\
\hline Mean (SD) & $84.8(11.2)$ & $83.7(22.2)$ \\
\hline \multicolumn{3}{|l|}{$\mathrm{BMI}, \mathrm{kg} / \mathrm{m}^{2}$} \\
\hline Mean (SD) & $27.3(3.0)$ & $27.3(5.5)$ \\
\hline \multicolumn{3}{|c|}{ Letter digit substitution task, ${ }^{14}$ number correct } \\
\hline Mean (SD) & $43.9(11.5)$ & $43.4(10.0)$ \\
\hline \multicolumn{3}{|c|}{ SF-36 Physical Component Scale, ${ }^{25,26}$ scale $0-100$} \\
\hline Mean (SD) & $44.7(8.2)$ & $47.0(7.8)$ \\
\hline
\end{tabular}

WHO: World Health Organization; MET: metabolic equivalent of task; BMI: body mass index; SF: short form.

exercise programme as good or excellent, and four as moderately/sufficiently satisfactory. The majority evaluated the build-up, intensity and length of the exercise sessions as good (Table 2). A total of 17 patients reported that they intended to continue their training after study completion although 9 of 
Table 2. Post-intervention ratings of physical exercise programme, training aids and home-based guidance.

\begin{tabular}{llll}
\hline Patient rating of difficulty/quantity & (Too) Easy/little & Just right & (Too) Difficult/many \\
\hline Build-up of the programme & $3(15 \%)$ & $14(70 \%)$ & $3(15 \%)$ \\
Number of exercises per week & $0(0 \%)$ & $11(55 \%)$ & $9(45 \%)$ \\
Intensity of sessions & $3(15 \%)$ & $15(75 \%)$ & $2(10 \%)$ \\
Session duration & $3(15 \%)$ & $14(70 \%)$ & $3(15 \%)$ \\
Difficulty Polar website & $1(5 \%)$ & $17(85 \%)$ & $2(10 \%)$ \\
Difficulty Polar heart rate watch & $0(0 \%)$ & $17(85 \%)$ & $3(15 \%)$ \\
\hline Patient rating of quality & Excellent/good & Sufficient & Insufficient/poor \\
\hline Choice of activities & $13(68 \%)$ & $5(26 \%)$ & $1(5 \%)$ \\
Experience of individualized training & $14(74 \%)$ & $4(21 \%)$ & $1(5 \%)$ \\
E-mail contact with physical therapist & $16(84 \%)$ & $2(11 \%)$ & $1(5 \%)$ \\
Involvement of physical therapist & $18(95 \%)$ & $1(5 \%)$ & $0(0 \%)$ \\
Final programme rating & $16(84 \%)$ & $2(11 \%)$ & $1(5 \%)$ \\
\hline
\end{tabular}

them intended to decrease the frequency to one or two training sessions per week.

Overall, the difficulty of the training aids appeared to be acceptable. The remotely supervised character of the programme, the contact with the physiotherapist by e-mail and the involvement of the physiotherapist were highly valued. However, three patients indicated that they would have preferred group training.

Some quotes of patients extracted from their e-mails to the physiotherapist are displayed in the Online Box.

\section{Feasibility of neuropsychological and physical assessments}

Administration of the neuropsychological tests at the patients' home took about 100 minutes. Estimated time to complete the questionnaires was about 60 minutes. There were (logistical) problems with planning the baseline exercise test, explaining a large range of 6-75 days between these assessments in some cases, although in $91 \%$ of patients, this interval was shorter than 50 days, with a median of 20 days.

At follow-up, one exercise group patient did not return questionnaires, whereas all control group patients returned completed questionnaires. Individual patient circumstances and logistical problems with the exercise test explained intervals ranging from -35 (exercise test preceding neuropsychological assessment) to 62 days between follow-up neuropsychological and physical assessments, although in $90 \%$ of patients this interval ranged from -6 to 18 days, with a median of 4 days.

\section{Objective physical outcomes and self- reported physical activity}

Table 3 shows the baseline, follow-up and change scores with respect to absolute and relative $\mathrm{VO}_{2}$ peak, BMI and self-reported activity for both groups as well as between-group differences in these outcomes. In short, after six months, mean absolute $\mathrm{VO}_{2}$ peak (the primary outcome in these analyses) in the exercise group improved $6.0 \%$ compared to baseline, and mean relative $\mathrm{VO}_{2}$ peak improved $7.3 \%$. There were substantial inter-individual differences in absolute $\mathrm{VO}_{2}$ peak, with the largest improvement $26.4 \%$, and the largest decline $18.1 \%$. Two of the four patients with a decline had a substantially shorter intervention duration (see Online Table). The control group $(N=9)$ showed an average decline of $1.1 \%$ in absolute $\mathrm{VO}_{2}$ peak over time and no change in mean relative $\mathrm{VO}_{2}$ peak. However, three patients showed an absolute $\mathrm{VO}_{2}$ peak improvement $(2.5 \%, 9.0 \%$ and $17 \%)$. Betweengroup analyses at six months indicated that, after correction for baseline values, participants in the 


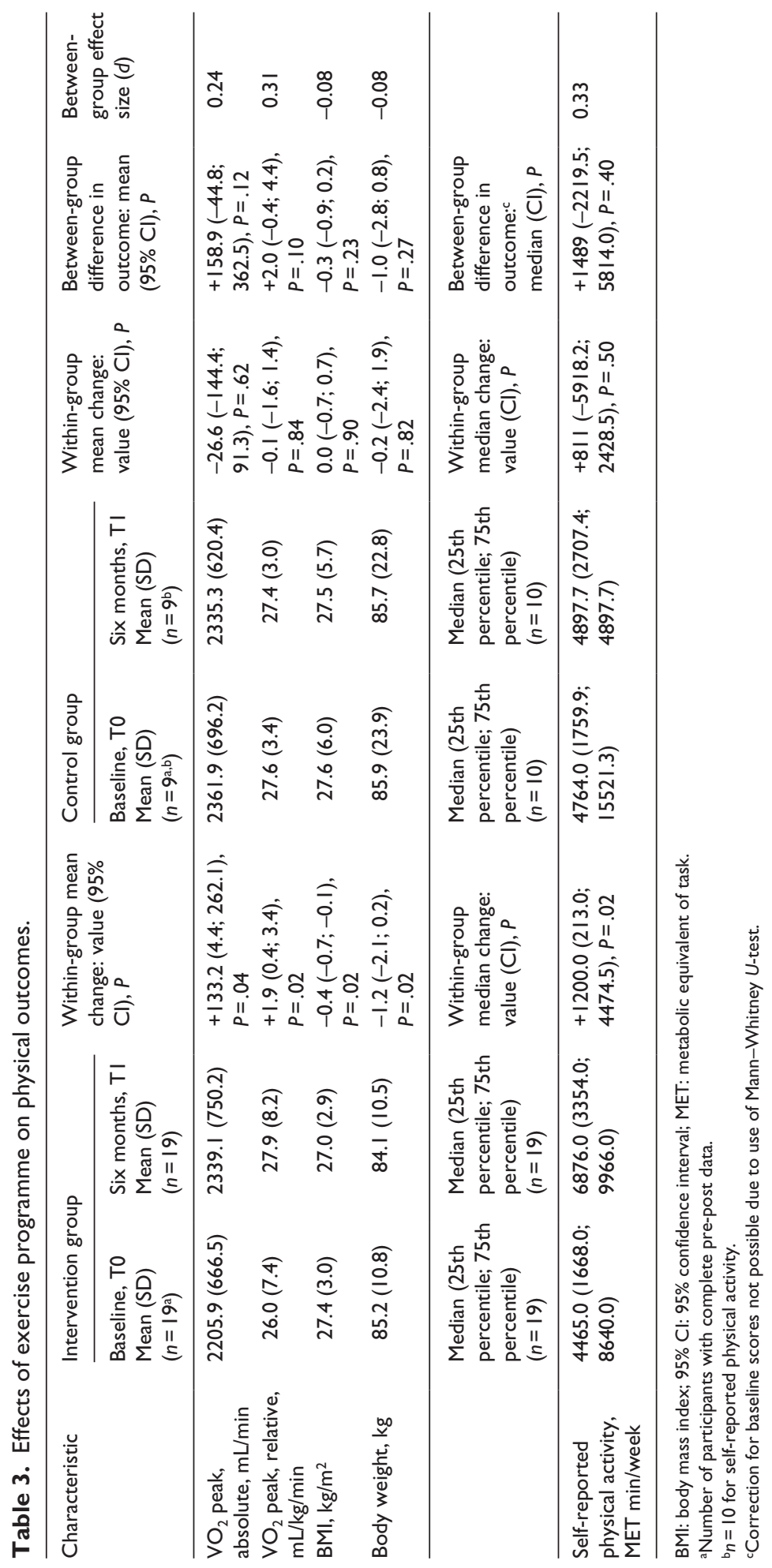


exercise group had a higher aerobic fitness compared to the control group $(+158.9 \mathrm{~mL} / \mathrm{min} ; 95 \%$ CI: 44.8 to 362.5 , and $2.0 \mathrm{~mL} / \mathrm{kg} / \mathrm{min} ; 95 \% \mathrm{CI}:-0.4$ to 4.4$)$, with a small effect size $(d=0.24)$.

At baseline, 19 of 23 patients $(83 \%)$ were classified as overweight, of whom 6 were obese. BMI decreased in the exercise group, but not in the control group, but there were no between-group differences in mean BMI at six months, after correction for baseline.

Self-reported physical activity varied widely among participants. The median self-reported physical activity increased in both groups, but more strongly in the exercise group. The average increase was $126 \%$ in the exercise group compared to $23 \%$ in the control group.

\section{Discussion}

The results of this pilot RCT provide support for the feasibility of a novel, remotely supervised, home-based aerobic exercise intervention for motivated patients with stable grades II and III gliomas. However, accrual of patients to the study was limited. The results suggest a positive effect of the programme on physical fitness. There were no adverse events related to the programme.

Overall, adherence was good; on average, participants in the exercise group adhered to $79 \%$ of the prescribed sessions (i.e. a mean of 2.4 sessions and an average training time of 126 minutes per week) and their experiences were positive although $45 \%$ reported that the frequency of the exercise sessions was (too) high. The programme included several features that are known to enhance treatment fidelity and enjoyment, and help overcome potential barriers to exercise (such as travel distance, feelings of uncertainty, motivational problems or time constraints). First, patients could exercise at home. Second, patients received regular guidance from both technology (a commercially available heart rate watch) and a physiotherapist. Remote contact is recommended in the literature as a strategy to enhance adherence to online interventions. ${ }^{27-29}$ In previous studies of older adults, adherence to and effectiveness of blended care physical activity programmes were comparable to those observed in supervised onsite programmes. Third, patients were allowed, within certain limits, to choose their own activities. Current evidence suggests that exercise enjoyment is an important determinant of physical activity and exercise self-efficacy. ${ }^{30,31}$ These elements are aimed at implementation of regular exercise in patients' daily routines and may aid continuing to exercise after the intervention period had ended. ${ }^{29,32}$ In fact, 17 of 20 of our patients reported a willingness to continue the physical exercise programme after the study period although nine intended to decrease their schedule to 1 or 2 training sessions per week. Two sessions per week may be sufficient to maintain some of the beneficial effects of exercise,,$^{33}$ but it is below the general exercise recommendation for cancer survivors. ${ }^{34}$

The high level of feasibility in terms of adherence to the exercise programme observed among participants contrasted with the problems experienced in recruiting patients into the study. Initially, our aim was to include 60 patients, but we were only able to recruit 34 . In all, $47 \%$ of the 62 patients for whom a reason for declining participation was known indicated they lacked exercise motivation or time and probably considered this long and intensive programme too much for them. This number may actually be larger, as it is likely that some of the patients who did not provide a reason for declining to participate or did not respond to the invitation had similar reasons. It is difficult to determine in a randomized setting the extent to which patients decline participation because of a lack of interest in the intervention being offered, not wanting to complete questionnaires and tests, not wanting to be randomized or a combination of these factors. We would note that previous exercise studies in cancer patients also suffered from recruitment problems. In a meta-analysis of the effectiveness of exercise interventions for fatigue in cancer patients, only 10 of the 26 studies that conducted a sample size calculation were successful in recruiting their target. ${ }^{35}$

Although three-quarters of the participants had (had) symptoms of epilepsy, this did not complicate exercise adherence, except for one patient with very frequent seizures, for whom the programme was adapted slightly. Previous studies have suggested that exercise in patients with epilepsy may result in 
reduction of seizure susceptibility, improvement of quality of life, reduction of anxiety and depression, and better social integration. ${ }^{36}$

We also included outcome data on the intermediate (exercise) endpoint as a measure of feasibility of an exercise programme designed to improve cognitive function through improvements in $\mathrm{VO}_{2}$ peak in this specific population. As changes in $\mathrm{VO}_{2}$ peak can be better understood in comparison to patients who did not exercise, we included the comparison for this outcome in our analyses. The preliminary results suggest that the programme could be effective in increasing physical fitness and, perhaps, decreasing (over)weight. Absolute $\mathrm{VO}_{2}$ peak, as determined with maximum exercise testing, of patients in the exercise group increased significantly, as compared to no change in the control group. Although the study was not powered for statistical analysis on group differences in outcome, in this analysis a small but clinically relevant effect ${ }^{37,38}$ on absolute $\mathrm{VO}_{2}$ peak (a difference of $158.9 \mathrm{~mL} / \mathrm{min}$ ) was observed, which was comparable to the within-group analyses.

Self-reported physical activity varied widely among participants. Both groups in our study reported an increase in physical activity although the increase was substantially larger in the exercise group. This suggests a certain level of study reactivity (i.e. that patients in the control group also changed their behaviour, simply because they were study participants), which may have led to an underestimation of the observed intervention effects. This is a common problem in exercise oncology trials. ${ }^{39}$

Feasibility of administration of largely the same battery of neuropsychological tests and questionnaires that we used here was demonstrated in a previous large $\mathrm{RCT}^{21}$ in patients with stable glioma. In this study, patients experienced the exercise tests as more burdensome than the neuropsychological tests due to their physically challenging nature and because they had to travel.

An important limitation of our study is its small sample size. By allocation of participants in a 2:1 ratio, we could make optimum use of the number of participants, as it allowed us to evaluate feasibility and potential (within-group) effects of the programme in a larger group, but at the cost of limiting the precision of the between-group comparison.
In addition, as discussed above, uptake was low $(25 \%)$, predominantly due to patients' lack of time or motivation to participate in the trial and/or training programme. Overall, this resulted in a motivated group of participants for whom an intensive exercise programme may be particularly feasible. This suggests that the intervention, in its current form, may be of interest to a smaller subset of the target population.

Furthermore, all patients were tested on a cycle ergometer because group allocation and preferred training modality were not known until after baseline exercise testing. However, not everyone trained on a bicycle, and consequently both the baseline and follow-up tests lacked exercise specificity. This could have led to an underestimation of changes in aerobic fitness in patients who chose running or swimming as training modality.

Despite these limitations, the observed intervention effect and its specific relationship with programme adherence in this study warrant replication in a larger trial. The design of future exercise interventions and (randomized controlled) trials can hopefully benefit from the lessons we learned from this pilot study. First, we recommend to take the challenging recruitment in this patient group into account when allocating time for the recruitment phase of the study. Second, future trials could benefit from using objective measures of physical activity (i.e. accelerometers) instead of relying on self-report measures, which are prone to overestimation of physical activity levels. ${ }^{40,41}$ In line with the literature, ${ }^{42}$ we recommend the use of cardiopulmonary exercise testing for baseline testing in order to minimize risk of exercise-related adverse events (in this study, we excluded one patient at risk). This maximum exercise test is considered the gold standard for assessment of cardiorespiratory fitness because it provides the most accurate determination of peak oxygen uptake $\left(\mathrm{VO}_{2}\right.$ peak). ${ }^{42}$ Future trials might employ study logistics to enable adaptation of type of exercise test to training activities. The results can also be used in prescribing individual exercise programmes (and their reliable evaluation in case of additional follow-up exercise testing). Finally, studies offering a programme with a lower frequency of exercise activities per week 
might be less likely to suffer from problems with uptake and may have a higher programme adherence than our study. Although two sessions per week is below the general exercise recommendation for cancer survivors, ${ }^{34}$ patients with low physical fitness would likely still benefit from two sessions per week. During the programme, the training frequency could be increased for patients who are able and willing, thus providing even better tailoring to individual needs and abilities.

The primary aim of our study was to investigate the feasibility of an aerobic exercise intervention for the improvement of cognitive function through improvement of aerobic fitness, as it has been suggested that increased cardiorespiratory fitness (i.e. $\mathrm{VO}_{2}$ peak) may be associated with improvements in cognitive capacity. ${ }^{43,44}$ The preliminary results presented here suggest that this intensive exercise programme helped to improve cardiorespiratory fitness. A forthcoming evaluation of the study results will address the question whether our physical exercise programme also had an effect on cognitive functioning in this patient population. This will include detailed investigation of the effects of the exercise programme on the domains of attention, memory and executive function, and on selfreported cognitive symptoms, fatigue, sleep, mood and quality life.

Given the need for management of the multitude of symptoms in this neuro-oncological patient group, and the potential applicability of exercise to aid in this management, ${ }^{2}$ the findings discussed here warrant further investigation of exercise interventions that may benefit this population.

\section{Clinical messages}

- Six months of home-based, remotely coached exercise is feasible in a select group of motivated glioma patients.

- Recruitment of the total group of stable patients to a long and intensive exercise intervention is difficult.

- Exercise may improve cardiorespiratory fitness and physical functioning in neurooncological patients.

\section{Acknowledgements}

We thank our research assistants/technicians: Eva Visser, MSc; Karin Eichhorn, MSc; Fleur Franken, MSc; Sophie van der Linden, MSc; Wietske Schimmel, MSc; Eline Verhaak, MSc, as well as (the investigators of) the participating centres: Medical Center Haaglanden, The Hague; Elisabeth-TweeSteden Hospital, Tilburg; Erasmus Medical Center, Rotterdam; and the Sports Medical Centers from the Amphia Hospital Breda, Elkerliek Hospital Helmond, Jeroen Bosch Hospital Den Bosch, Maxima Medical Center Eindhoven, Medical Center Haaglanden Den Haag, SportMedisch Advies Centrum Rotterdam, The Netherlands.

\section{Declaration of Conflicting Interests}

The author(s) declared no potential conflicts of interest with respect to the research, authorship and/or publication of this article.

\section{Ethical approval}

All procedures performed in studies involving human participants were in accordance with the ethical standards of the institutional and/or national research committee and with the 1964 Helsinki declaration and its later amendments or comparable ethical standards.

\section{Funding}

The author(s) disclosed receipt of the following financial support for the research, authorship and/or publication of this article: This research was supported by a grant from the Dutch Cancer Society (UvT2010-4642). LWJ is supported by grants from the National Cancer Institute, AKTIV Against Cancer, Kavli Trust, and the Memorial Sloan Kettering Cancer Center Support Grant/Core Grant (P30 CA008748).

\section{References}

1. Klein M, Duffau H and De Witt Hamer PC. Cognition and resective surgery for diffuse infiltrative glioma: an overview. J Neurooncol 2012; 108(2): 309-318.

2. Cormie P, Nowak AK, Chambers SK, et al. The potential role of exercise in neuro-oncology. Front Oncol 2015; 5: 85 .

3. Capozzi LC, Boldt KR, Easaw J, et al. Evaluating a 12-week exercise program for brain cancer patients. Psychooncology 2016; 25(3): 354-358.

4. Jones LW, Guill B, Keir ST, et al. Exercise interest and preferences among patients diagnosed with primary brain cancer. Support Care Cancer 2007; 15(1): $47-55$. 
5. Nicole Culos-Reed S, Leach HJ, Capozzi LC, et al. Exercise preferences and associations between fitness parameters, physical activity, and quality of life in highgrade glioma patients. Support Care Cancer 2016; 25: 1237-1246.

6. Hillman CH, Erickson KI and Kramer AF. Be smart, exercise your heart: exercise effects on brain and cognition. Nat Rev Neurosci 2008; 9(1): 58-65.

7. Lautenschlager NT, Cox K and Cyarto EV. The influence of exercise on brain aging and dementia. Biochim Biophys Acta 2012; 1822(3): 474-481.

8. Hotting $\mathrm{K}$ and Roder B. Beneficial effects of physical exercise on neuroplasticity and cognition. Neurosci Biobehav R 2013; 37(9PtB): 2243-2257.

9. Long BJ, Calfas KJ, Wooten W, et al. A multisite field test of the acceptability of physical activity counseling in primary care: project PACE. Am J Prev Med 1996; 12(2): 73-81.

10. Vos JA. Ergometrie En Trainingsbegeleiding [Ergometry and training supervision]. 7th ed. Otterlo, The Netherlands, 2013.

11. West MA, Parry M, Asher R, et al. The effect of betablockade on objectively measured physical fitness in patients with abdominal aortic aneurysms - a blinded interventional study. Br J Anaesth 2015; 114(6): 878-885.

12. Thomas S, Reading $J$ and Shephard RJ. Revision of the physical activity readiness questionnaire (PAR-Q). Can J Sport Sci 1992; 17(4): 338-345.

13. Scott NW, McPherson GC, Ramsay CR, et al. The method of minimization for allocation to clinical trials. A review. Control Clin Trials 2002; 23(6): 662-674.

14. Jolles J, Houx PJ, Van Boxtel MPJ, et al. Maastricht aging study: determinants of cognitive aging. Maastricht, The Netherlands: Neuropsych Publishers, 1995.

15. ALEA. ALEA AVL, The Netherlands, https://prod. tenalea.net/avl/dm/

16. Polar. Polar personal trainer, https://www.polarpersonaltrainer.com/

17. Kemper HGC, Ooijendijk WTM and Stiggelbout M. Consensus over de Nederlandse Norm voor Gezond Bewegen [Consensus on the Dutch standard for healthy exercise]. TSG 2000; 78(3): 180-183.

18. IPAQ-group. IPAQ scoring protocol, https://sites.google. com/site/theipaq/scoring-protocol

19. Craig CL, Marshall AL, Sjostrom M, et al. International physical activity questionnaire: 12-country reliability and validity. Med Sci Sport Exer 2003; 35(8): 1381-1395.

20. Pescatello LS. ACSM's guidelines for exercise testing and prescription. 9th ed. Philadelphia, PA: Wolters Kluwer/ Lippincott Williams \& Wilkins, 2014.

21. Gehring K, Sitskoorn MM, Gundy CM, et al. Cognitive rehabilitation in patients with gliomas: a randomized, controlled trial. J Clin Oncol 2009; 27(22): 3712-3722.

22. Cohen J. Statistical power analysis for the behavioral sciences. Hillsdale, NJ: Lawrence Erlbaum Associates, 1988.
23. Corp I. IBM SPSS statistics for windows. 22nd ed. Armonk, NY: IVBM Corp., 2013.

24. Team RC. R: a language and environment for statistical computing. Vienna: R Foundation for Statistical Computing, 2016.

25. Ware JE Jr and Sherbourne CD. The MOS 36-item shortform health survey (SF-36). I. Conceptual framework and item selection. Medcare 1992; 30(6): 473-483.

26. Aaronson NK, Muller M, Cohen PD, et al. Translation, validation, and norming of the Dutch language version of the SF-36 Health Survey in community and chronic disease populations. J Clin Epidemiol 1998; 51(11): 1055-1068.

27. Beatty L and Binnion C. A systematic review of predictors of, and reasons for, adherence to online psychological interventions. Int J Behav Med 2016; 23: 776-794.

28. Bluethmann SM, Vernon SW, Gabriel KP, et al. Taking the next step: a systematic review and meta-analysis of physical activity and behavior change interventions in recent post-treatment breast cancer survivors. Breast Cancer Res Treat 2015; 149(2): 331-342.

29. Geraedts H, Zijlstra A, Bulstra SK, et al. Effects of remote feedback in home-based physical activity interventions for older adults: a systematic review. Patient Educ Couns 2013; 91(1): 14-24.

30. Ungar N, Wiskemann J and Sieverding M. Physical activity enjoyment and self-efficacy as predictors of cancer patients' physical activity level. Front Psychol 2016; 7: 898.

31. Valenzuela T, Okubo Y, Woodbury A, et al. Adherence to technology-based exercise programs in older adults: a systematic review. J Geriatr Phys Ther. Epub ahead of print 29 June 2016. DOI: 10.1519/JPT.000000000 0000095.

32. Ashworth NL, Chad KE, Harrison EL, et al. Home versus center based physical activity programs in older adults. Cochrane Database Syst Rev 2005(1): CD004017.

33. Wenger HA and Bell GJ. The interactions of intensity, frequency and duration of exercise training in altering cardiorespiratory fitness. Sports Med 1986; 3(5): 346-356.

34. Schmitz KH, Courneya KS, Matthews C, et al. American College of Sports Medicine roundtable on exercise guidelines for cancer survivors. Med Sci Sport Exer 2010; 42(7): 1409-1426.

35. Cramp F and Byron-Daniel J. Exercise for the management of cancer-related fatigue in adults. Cochrane Database Syst Rev 2012; 11: CD006145.

36. Arida RM, Scorza FA, Gomes da Silva S, et al. The potential role of physical exercise in the treatment of epilepsy. Epilepsy Behav 2010; 17(4): 432-435.

37. Swank AM, Horton J, Fleg JL, et al. Modest increase in peak VO2 is related to better clinical outcomes in chronic heart failure patients: results from heart failure and a controlled trial to investigate outcomes of exercise training. Circ Heart Fail 2012; 5(5): 579-585. 
38. Ainsworth BE, Haskell WL, Herrmann SD, et al. 2011 compendium of physical activities: a second update of codes and MET values. Med Sci Sport Exer 2011; 43(8): 1575-1581.

39. Steins Bisschop CN, Courneya KS, Velthuis MJ, et al. Control group design, contamination and drop-out in exercise oncology trials: a systematic review. PLOS ONE 2015; 10(3): e0120996.

40. Van Poppel MN, Chinapaw MJ, Mokkink LB, et al. Physical activity questionnaires for adults: a systematic review of measurement properties. Sports Med 2010; 40(7): 565-600.

41. Harris TJ, Owen CG, Victor CR, et al. A comparison of questionnaire, accelerometer, and pedometer: measures in older people. Med Sci Sport Exer 2009; 41(7): 1392-1402.

42. Jones LW, Eves ND, Haykowsky M, et al. Cardiorespiratory exercise testing in clinical oncology research: systematic review and practice recommendations. Lancet Oncol 2008; 9(8): 757-765.

43. Colcombe $\mathrm{S}$ and Kramer AF. Fitness effects on the cognitive function of older adults: a meta-analytic study. Psychol Sci 2003; 14(2): 125-130.

44. Smith PJ, Blumenthal JA, Hoffman BM, et al. Aerobic exercise and neurocognitive performance: a meta-analytic review of randomized controlled trials. Psychosom Med 2010; 72(3): 239-252. 